\title{
The spectrum of an asymmetric annihilation process
}

\author{
Arvind Ayyer ${ }^{1}$ and Volker Strehl ${ }^{2}$ \\ ${ }^{1}$ Institut de Physique Théorique, C. E. A. Saclay, 91191 Gif-sur-Yvette Cedex, France \\ ${ }^{2}$ Department of Computer Science, Universität Erlangen-Nürnberg, Haberstrasse 2, D-91058 Erlangen, Germany
}

\begin{abstract}
In recent work on nonequilibrium statistical physics, a certain Markovian exclusion model called an asymmetric annihilation process was studied by Ayyer and Mallick. In it they gave a precise conjecture for the eigenvalues (along with the multiplicities) of the transition matrix. They further conjectured that to each eigenvalue, there corresponds only one eigenvector. We prove the first of these conjectures by generalizing the original Markov matrix by introducing extra parameters, explicitly calculating its eigenvalues, and showing that the new matrix reduces to the original one by a suitable specialization. In addition, we outline a derivation of the partition function in the generalized model, which also reduces to the one obtained by Ayyer and Mallick in the original model.

Résumé. Dans un travail récent sur la physique statistique hors équilibre, un certain modèle d'exclusion Markovien appelé "processus d'annihilation asymétriques" a été étudié par Ayyer et Mallick. Dans ce document, ils ont donné une conjecture précise pour les valeurs propres (avec les multiplicités) de la matrice stochastique. Ils ont en outre supposé que, pour chaque valeur propre, correspond un seul vecteur propre. Nous prouvons la première de ces conjectures en généralisant la matrice originale de Markov par l'introduction de paramètres supplémentaires, calculant explicitement ses valeurs propres, et en montrant que la nouvelle matrice se réduit à l'originale par une spécialisation appropriée. En outre, nous présentons un calcul de la fonction de partition dans le modèle généralisé, ce qui réduit également à celle obtenue par Ayyer et Mallick dans le modèle original.
\end{abstract}

Keywords: Reaction diffusion process, non-equilibrium lattice model, transfer matrix Ansatz, partition function, characteristic polynomial, Hadamard transform.

\section{Introduction}

In the past few years, special stochastic models motivated by nonequilibrium statistical mechanics have motivated several combinatorial problems. The most widely studied problem among these has been the totally asymmetric simple exclusion process (TASEP). The model is defined on a one dimensional lattice of $L$ sites, each site of which either contains a particle or not. Particles in the interior try to jump with rate 1 to the site to the right. The jump succeeds if that site is empty and fails if not. On the boundary, particles enter with rate $\alpha$ on the first site if it is empty and leave from the last site with rate $\beta$. This was first solved in 1993 by developing a new technique now called the matrix product representation [1].

It was initially studied in a combinatorial setting by Shapiro and Zeilberger in an almost forgotten paper [3] in 1982, but only after the steady state distribution of the model was explicitly presented in 
[1], the problem gained widespread attention. One of the reasons for this interest was that the common denominator of the steady state probabilities for a system of size $L$ was $C_{L+1}$, the $(L+1)$-th Catalan number. One of the first articles to explain this fact combinatorially was the one by Duchi and Schaeffer [4], who enlarged the space of configurations to one in bijection with bicolored Motzkin paths and showed that the steady state distribution was uniform on this space. The analogous construction for the partially asymmetric version of the model (PASEP) has been done in [5].

Further work has been on the relationship of the total and partially asymmetric exclusion processes to different kinds of tableaux by Corteel and Williams [6, 7, 8] (permutation tableaux, staircase tableaux) and by Viennot [9] (Catalan tableaux), to lattice paths [10], and to Askey-Wilson polynomials [7].

Just like the common denominator for the TASEP of size $L$ was the Catalan number $C_{L}$ (which has many combinatorial interpretations), the common denominator for the asymmetric annihilation process considered in [2] in a system of size $L$ at $\alpha=1 / 2, \beta=1$ is $2^{\left(\begin{array}{c}(+1 \\ 2\end{array}\right)}$ which is the number of domino tilings of an Aztec diamond of size $L$ as well as the number of 2-enumerated $L \times L$ alternating sign matrices. One can therefore hope to enlarge the configuration space as was done for the TASEP [4] to explain this phenomena.

The remainder of this extended abstract is organized as follows: In Sec. 2 we describe the model of the asymmetric annihilation process. In Sec. 3 we present some of the main results obtained by Ayyer and Mallick in [2]. Their work lead to a conjecture about the spectrum of this process. In Sec. 4 we prove this conjecture by appropriately extending the model and viewing it in a different basis obtained by a variant of the Hadamard transform. In the concluding section we outline the derivation of the partition function for the generalized model using the same transformation, an approach very different from the way Ayyer and Mallick obtained the partition function in the original model.

\section{The model}

Motivated by Glauber dynamics of the Ising model, Ayyer and Mallick [2] considered a non-equilibrium system on a finite lattice with $L$ sites labelled from 1 to $L$. States of the system are encoded by bitvectors $\boldsymbol{b}=b_{1} b_{2} \ldots b_{L}$ of length $L$, where $b_{j} \in \mathbb{B}=\{0,1\}$, so that we have a total of $2^{L}$ states. These bit vectors may be represented numerically using the binary expansion $(\boldsymbol{b})_{2}=b_{L}+b_{L-1} 2^{1}+b_{L-2} 2^{2}+\cdots+b_{1} 2^{L-1}$, which introduces a total order on $\mathbb{B}^{L}$, so that we shall write $\boldsymbol{b}<\boldsymbol{c}$ iff $(\boldsymbol{b})_{2}<(\boldsymbol{c})_{2}$. All matrices and vectors are indexed w.r.t. this order.

The evolution rules of the system introduced in [2] can now be stated as rewrite rules for bit vectors:

- In the bulk we have right shift and annihilation given by

$$
\begin{array}{lll}
\text { right shift } & 10 \rightarrow 01 \quad \text { with rate } 1, \\
\text { annihilation } & 11 \rightarrow 00 & \text { with rate } \lambda,
\end{array}
$$

and visualized (for $L=8$ ) in Fig. 1 .
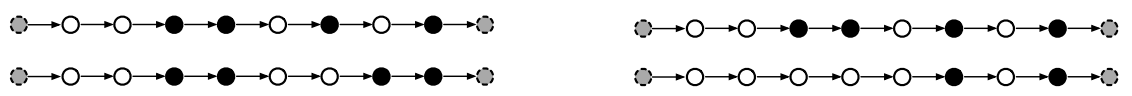

Fig. 1: Right shift $00110 \underline{101} \rightarrow 00110 \underline{011}$ and annihilation $00 \underline{110101} \rightarrow 00 \underline{000101}$ 
- On the left boundary, particles enter by left creation in a way consistent with the bulk dynamics. A particle at site 1 may also be left annihilated (due to a virtual particle at site 0 ). Therefore, the first site evolves as

$$
\begin{array}{lll}
\text { left creation } & 0 \rightarrow 1 \quad \text { with rate } \alpha, \\
\text { left annihilation } & 1 \rightarrow 0 & \text { with rate } \alpha \lambda,
\end{array}
$$

as illustrated Fig. 2
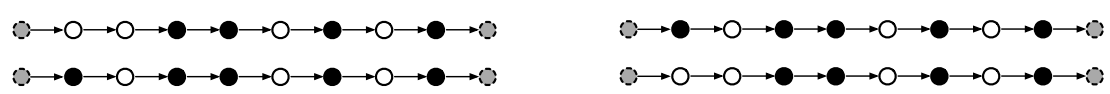

Fig. 2: Left creation $\underline{0} 0110101 \rightarrow \underline{10110011}$ and left annihilation $\underline{10110101} \rightarrow \underline{0} 0110101$

- Particles can exit from the last site by right annihilation (with a virtual particle at site $L+1$ ) according to right annihilation $\quad 1 \rightarrow 0 \quad$ with rate $\beta$,

as illustrated by Fig. 3

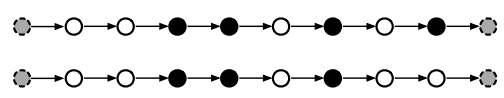

Fig. 3: Right annihilation $0011010 \underline{1} \rightarrow 1011001 \underline{0}$

Note that all transition rules except left creation are monotonically decreasing w.r.t. the natural order of bit vectors. Thus the transition matrix, as discussed in the next section, is not in triangular shape.

Following [2], we will take $\lambda=1$ as that is the only case for which they derive explicit formulae.

\section{Algebraic properties of the model}

Is this section we present without proofs the main results as obtained by Ayyer and Mallick in [2]. First recall the general concept:

Definition 1 A (continuous-time) transition matrix or Markov matrix or stochastic matrix is a square matrix of size equal to the cardinality of the configuration space whose $(i, j)$-th entry is given by the rate of the transition from configuration $j$ to configuration $i$, when $i$ is not equal to $j$. The $(i, i)$-th entry is then fixed by demanding that the entries in each column sum to zero.

The Markov chain we defined in the previous section satisfies what [2] call the "transfer matrix Ansatz". The following general definition applies to any family of Markov processes defined by Markov matrices $\left\{M_{L}\right\}$ of increasing sizes (in most physical applications, $L$ is the size of the system).

Definition 2 A family $M_{L}$ of Markov processes satisfies the Transfer Matrix Ansatz if there exist matrices $T_{L, L+1}$ for all sizes $L$ such that

$$
\text { (TMA) } \quad M_{L+1} T_{L, L+1}=T_{L, L+1} M_{L} .
$$

We also impose that this equality is nontrivial in the sense that $M_{L+1} T_{L, L+1} \neq 0$. 
The rectangular transfer matrices $T_{L, L+1}$ can be interpreted as semi-similarity transformations connecting Markov matrices of different sizes.

The last condition is important because there is always a trivial solution whenever we are guaranteed a unique Perron-Frobenius eigenvector for all transition matrices $M_{L}$. If $\left|v_{L}\right\rangle$ is this eigenvector of $M_{L}$ and $\left\langle 1_{L}\right|=(1,1, \ldots, 1)$, the matrix $V_{L, L+1}=\left|v_{L+1}\right\rangle\left\langle 1_{L}\right|$ satisfies (TMA) since the Markov matrices satisfy the conditions $\left\langle 1_{L}\right| M_{L}=0$ and $M_{L+1}\left|v_{L+1}\right\rangle=0$.

The above definition leads immediately to a recursive computation of the steady state vector, which is the zero eigenvector. First we have

$$
0=T_{L, L+1} M_{L}\left|v_{L}\right\rangle=M_{L+1} T_{L, L+1}\left|v_{L}\right\rangle,
$$

which, assuming $T_{L, L+1}\left|v_{L}\right\rangle \neq 0$, and taking into account the uniqueness of the steady state, allows us to define $\left|v_{L+1}\right\rangle$ so that

$$
T_{L, L+1}\left|v_{L}\right\rangle=\left|v_{L+1}\right\rangle .
$$

This is very analogous to the matrix product representation of [1] because the steady state probability of any configuration of length $L+1$ is expressed as a linear combination of those of length $L$. The transfer matrix Ansatz is a stronger requirement than the matrix product representation in the sense that not every system which admits the representation satisfies the Ansatz. For example, the only solution for (TMA) in the case of the TASEP is the trivial one.

For our system introduced above, the Markov matrices $M_{L}$ are of size $2^{L}$. As mentioned, the entries of these matrices are indexed w.r.t. the naturally ordered basis of binary vectors of length $L$. For convenience, here are the first three of these matrices:

$$
M_{1}=\left[\begin{array}{cc}
-\alpha & \alpha+\beta \\
\alpha & -\alpha-\beta
\end{array}\right], M_{2}=\left[\begin{array}{cccc}
\star & \beta & \alpha & 1 \\
0 & \star & 1 & \alpha \\
\alpha & 0 & \star & \beta \\
0 & \alpha & 0 & \star
\end{array}\right], M_{3}=\left[\begin{array}{cccccccc}
\star & \beta & 0 & 1 & \alpha & 0 & 1 & 0 \\
0 & \star & 1 & 0 & 0 & \alpha & 0 & 1 \\
0 & 0 & \star & \beta & 1 & 0 & \alpha & 0 \\
0 & 0 & 0 & \star & 0 & 1 & 0 & \alpha \\
\alpha & 0 & 0 & 0 & \star & \beta & 0 & 1 \\
0 & \alpha & 0 & 0 & 0 & \star & 1 & 0 \\
0 & 0 & \alpha & 0 & 0 & 0 & \star & \beta \\
0 & 0 & 0 & \alpha & 0 & 0 & 0 & \star
\end{array}\right] .
$$

As for the diagonal elements $\star$, they have to be set such that the column sums vanish.

We now state without proof some important results on the Markov matrices of the system. These are proved in [2]. We first show that the Markov matrix itself satisfies a recursion of order one.

Theorem 1 Let $\sigma$ denote the matrix $\sigma=\left[\begin{array}{ll}0 & 1 \\ 1 & 0\end{array}\right]$, and $\mathbb{1}_{L}$ denote the identity matrix of size $2^{L}$. Then

$$
M_{L}=\left[\begin{array}{c|c}
M_{L-1}-\alpha\left(\sigma \otimes \mathbb{1}_{L-2}\right) & \alpha \mathbb{1}_{L-1}+\left(\sigma \otimes \mathbb{1}_{L-2}\right) \\
\hline \alpha \mathbb{1}_{L-1} & M_{L-1}-\mathbb{1}_{L-1}-\alpha\left(\sigma \otimes \mathbb{1}_{L-2}\right)
\end{array}\right],
$$

where $M_{L}$ is written as a $2 \times 2$ block matrix with each block made up of matrices of size $2^{L-1}$.

The transfer matrices can also be explicitly constructed by a recursion of order one. 
Theorem 2 There exist transfer matrices for the model. If one writes the transfer matrix from size $2^{L-1}$ to size $2^{L}$ by a block decomposition of matrices of size $2^{L-1} \times 2^{L-1}$ as

$$
\begin{gathered}
T_{L-1, L}=\left[\frac{T_{1}^{(L-1)}}{T_{2}^{(L-1)}}\right] \text {, then the matrix } T_{L, L+1} \text { can be written as } T_{L, L+1}=\left[\frac{T_{1}^{(L)}}{T_{2}^{(L)}}\right] \text {, with } \\
T_{1}^{(L)}=\left[\begin{array}{c|c}
T_{1}^{(L-1)}+\alpha^{-1} T_{2}^{(L-1)} & 2 T_{2}^{(L-1)}+\alpha^{-1} T_{2}^{(L-1)} \\
\hline\left(\sigma \otimes \mathbb{1}_{L-2}\right) T_{2}^{(L-1)} & \alpha^{-1} T_{2}^{(L-1)}
\end{array}\right], T_{2}^{(L)}=\left[\begin{array}{c|c}
2 T_{2}^{(L-1)} & T_{2}^{(L-1)}\left(\sigma \otimes \mathbb{1}_{L-2}\right) \\
\hline 0 & T_{2}^{(L-1)}
\end{array}\right] .
\end{gathered}
$$

This, along with the initial condition

$$
T_{1,2}=\left[\begin{array}{cc}
1+\beta+\alpha \beta & \alpha+\beta+\alpha \beta \\
\alpha & 1 \\
\alpha+\alpha \beta & \alpha \beta \\
0 & \alpha
\end{array}\right],
$$

determines recursively a family of transfer matrices for the matrices $M_{L}$.

We can also use the transfer matrices to calculate properties of the steady state distribution of the Markov process. One quantity of interest is the so called normalization factor or partition function.

Definition 3 Let the entries of the kernel $\left|v_{L}\right\rangle$ of $M_{L}$ be normalized so that their sum is 1 and each entry written in rationally reduced form. Then the partition function $Z_{L}$ for the system of size $L$ is the least common multiple of the denominators of the entries of $\left|v_{L}\right\rangle$.

Because of the way the transfer matrix has been constructed $Z_{L}$ is the sum of the entries in $v_{L}$. For example, the system of size one has $\left|v_{1}\right\rangle=\left[\begin{array}{c}\alpha+\beta \\ \alpha\end{array}\right]$, whence $Z_{1}=2 \alpha+\beta$.

Corollary 3 The partition function of the system of size $L$ is given by

$$
Z_{L}=2^{\left(\frac{L-1}{2}\right)}(1+2 \alpha)^{L-1}(1+\beta)^{L-1}(2 \alpha+\beta) .
$$

\section{Spectrum of the Markov matrices}

In this section, we consider the eigenvalues of the Markov matrices $M_{L}$ of the asymmetric annihilation process. The following result was stated as a conjecture in [2]. This will be a corollary of the main result (Theorem 8) of this article.

Theorem 4 Let the polynomials $A_{L}(x)$ and $B_{L}(x)$ be defined as

$$
A_{L}(x)=\prod_{k=0}^{\lceil L / 2\rceil}(x+2 k)^{\left(\begin{array}{c}
L-1 \\
2 k
\end{array}\right)}, \quad B_{L}(x)=\prod_{k=0}^{\lfloor L / 2\rfloor}(x+2 k+1)^{\left(\begin{array}{c}
L-1 \\
2 k+1
\end{array}\right)} .
$$

Then the characteristic polynomial $P_{L}(x)$ of $M_{L}$ is given by

$$
P_{L}(x)=A_{L}(x) A_{L}(x+2 \alpha+\beta) B_{L}(x+\beta) B_{L}(x+2 \alpha),
$$


and successive ratios of characteristic polynomials are given by

$$
\frac{P_{L+1}(x)}{P_{L}(x)}=B_{L}(x+1) B_{L}(x+2 \alpha+\beta+1) A_{L}(x+\beta+1) A_{L}(x+2 \alpha+1) .
$$

This gives only $2 L$ distinct eigenvalues out of a possible $2^{L}$. There is therefore the question of diagonalizability of the Markov matrix. Ayyer and Mallick [2] further conjecture the following.

Conjecture 1 The matrix $M_{L}$ is maximally degenerate in the sense that it has exactly $2 L$ eigenvectors.

For $L \geq 1$ we regard $\mathbb{B}^{L}$ as the vector space of bitvectors of length $L$ (over the binary field). The usual scalar product of vectors $\boldsymbol{b}, \boldsymbol{c} \in \mathbb{B}^{L}$ will be denoted by $\boldsymbol{b} \cdot \boldsymbol{c}$. We will take the set $V_{L}=\left\{|\boldsymbol{b}\rangle ; \boldsymbol{b} \in \mathbb{B}^{L}\right\}$ as the standard basis of a $2^{L}$-dimensional (real or complex) vector space, which we denote by $\mathcal{V}_{L}$. Indeed, we will consider $\mathcal{V}_{L}$ as a vector space over an extension over the real or complex field which contains all the variables that we introduce below. To be precise, we take $\mathcal{V}_{L}$ as a vector space over a field of rational functions which extends the real or complex field.

The following definitions of linear transformations, when considered as matrices, refer to this basis, if not stated otherwise. $\mathcal{V}_{L}$ is the $L$-th tensor power of the 2 -dimensional space $\mathcal{V}_{1}$ in an obvious way.

The transformation $\sigma$ of $\mathcal{V}_{1}$ is given by the matrix $\sigma=\left[\begin{array}{ll}0 & 1 \\ 1 & 0\end{array}\right]$ and this extends naturally to transformations $\sigma^{\boldsymbol{b}}$ of $\mathcal{V}_{L}$ for $\boldsymbol{b}=b_{1} b_{2} \ldots b_{L} \in \mathbb{B}^{L}$ :

$$
\sigma^{\boldsymbol{b}}=\sigma^{b_{1} b_{2} \ldots b_{L}}=\sigma^{b_{1}} \otimes \sigma^{b_{2}} \otimes \cdots \otimes \sigma^{b_{L}} .
$$

Definition 4 For a vector $\boldsymbol{\alpha}=\left(\alpha_{\boldsymbol{b}}\right)_{\boldsymbol{b} \in \mathbb{B}^{L}}$ of variables we define the transformation $\mathcal{A}_{L}(\boldsymbol{\alpha})$ of $\mathcal{V}_{L}$ as

$$
A_{L}(\boldsymbol{\alpha})=\sum_{\boldsymbol{b} \in \mathbb{B}^{L}} \alpha_{\boldsymbol{b}} \sigma^{\boldsymbol{b}}
$$

A direct way to define these matrices is $\left\langle\boldsymbol{b}\left|A_{L}\right| \boldsymbol{c}\right\rangle=\alpha_{\boldsymbol{b} \oplus \boldsymbol{c}},\left(\boldsymbol{b}, \boldsymbol{c} \in \mathbb{B}^{L}\right)$, where $\oplus$ denotes the component wise mod-2-addition (exor) of bit vectors.

For $1 \leq j \leq L$ we define the involutive mappings

$$
\phi_{j}: \mathbb{B}^{L} \rightarrow \mathbb{B}^{L}: b_{1} \ldots b_{j-1} b_{j} b_{j+1} \ldots b_{L} \mapsto \phi_{j} \boldsymbol{b}=b_{1} \ldots b_{j-1} \overline{b_{j}} b_{j+1} \ldots b_{L}
$$

by complementing the $j$-th component, and involutions

$$
\psi_{j}: \mathbb{B}^{L} \rightarrow \mathbb{B}^{L}: \boldsymbol{b} \mapsto \phi_{j} \phi_{j+1} \boldsymbol{b}
$$

by complementing components indexed $j$ and $j+1$, where $\psi_{L}$ is the same as $\phi_{L}$.

Definition $5 \quad$ 1. For $1 \leq j \leq L$ we define the projection operators $\mathcal{P}_{L, j}$ acting on $\mathcal{V}_{L}$ by

$$
\mathcal{P}_{L, j}=\sum_{\boldsymbol{b} \in \mathbb{B}^{L}}|\boldsymbol{b}\rangle\left\langle\boldsymbol{b}|-| \psi_{j}^{b_{j}}(\boldsymbol{b})\right\rangle\langle\boldsymbol{b}|
$$

2. For a vector $\boldsymbol{b}=\left(\beta_{1}, \beta_{2}, \ldots, \beta_{L}\right)$ of variables we put $\mathcal{B}_{L}(\boldsymbol{\beta})=\sum_{1 \leq j \leq L} \beta_{j} \mathcal{P}_{L, j}$. $B_{L}(\boldsymbol{\beta})$ denotes the matrix representing $\mathcal{B}_{L}(\boldsymbol{\beta})$ in the standard basis $V_{L}^{-}$. 
Note that in the sum for $\mathcal{P}_{L, j}$ only summands for which $b_{j}=1$, i.e., for which $\psi_{j}(\boldsymbol{b})<\boldsymbol{b}$, occur. Indeed: this condition allows only for two situations to contribute:

$$
\begin{aligned}
b_{j} b_{j+1}=10 & \mapsto \overline{b_{j}} \overline{b_{j+1}}=01 \\
b_{j} b_{j+1}=11 & \mapsto \overline{b_{j}} \overline{b_{j+1}}=00
\end{aligned} \quad \text { (annihilation) }
$$

Thus these operators $\psi_{j}$ encode the transitions of our model. Also note that by its very definition $B_{L}(\boldsymbol{\beta})$ is an upper triangular matrix.

Writing $\boldsymbol{\beta}=(\beta, \gamma, \delta)$ instead of $\left(\beta_{1}, \beta_{2}, \beta_{3}\right)$ we have for $L=3$

$$
B_{3}(\beta, \gamma, \delta)=\left[\begin{array}{cccccccc}
0 & -\delta & 0 & -\gamma & 0 & 0 & -\beta & 0 \\
0 & \delta & -\gamma & 0 & 0 & 0 & 0 & -\beta \\
0 & 0 & \gamma & -\delta & -\beta & 0 & 0 & 0 \\
0 & 0 & 0 & \delta+\gamma & 0 & -\beta & 0 & 0 \\
0 & 0 & 0 & 0 & \beta & -\delta & 0 & -\gamma \\
0 & 0 & 0 & 0 & 0 & \delta+\beta & -\gamma & 0 \\
0 & 0 & 0 & 0 & 0 & 0 & \gamma+\beta & -\delta \\
0 & 0 & 0 & 0 & 0 & 0 & 0 & \delta+\gamma+\beta
\end{array}\right] .
$$

Our main concern is now with the transformation given by

$$
\mathcal{M}_{L}(\boldsymbol{\alpha}, \boldsymbol{\beta})=\mathcal{A}_{L}(\boldsymbol{\alpha})-\mathcal{B}_{L}(\boldsymbol{\beta}) .
$$

Before we can state the main result we have to introduce some more notation, But before doing so, we note that the corresponding matrix $M_{L}(\boldsymbol{\alpha}, \boldsymbol{\beta})=A_{L}(\boldsymbol{\alpha})-B_{L}(\boldsymbol{\beta})$ reduces to the matrix $M_{L}$ above when properly specialized:

Lemma 5 We have $A_{L}\left(\boldsymbol{\alpha}^{\prime}\right)-B_{L}\left(\boldsymbol{\beta}^{\prime}\right)=M_{L}$ for $\boldsymbol{\alpha}^{\prime}=\left(\alpha_{\boldsymbol{b}}^{\prime}\right)_{\boldsymbol{b} \in \mathbb{B}^{L}}$ and $\boldsymbol{\beta}^{\prime}=\left(\beta_{j}^{\prime}\right)_{1 \leq j \leq L}$ given by

$$
\alpha_{\boldsymbol{b}}^{\prime}=\left\{\begin{array}{ll}
-\alpha & \text { if } \boldsymbol{b}=00 \ldots 00 \\
\alpha & \text { if } \boldsymbol{b}=10 \ldots 00 \\
0 & \text { otherwise }
\end{array} \text { and } \beta^{\prime}=\left\{\begin{array}{ll}
1 & \text { if } 1 \leq j<L \\
\beta & \text { if } j=L
\end{array} .\right.\right.
$$

We will now consider the transformation $\mathcal{M}_{L}(\boldsymbol{\alpha}, \boldsymbol{\beta})$ in a different basis of $\mathcal{V}_{L}$. Let $H=\frac{1}{\sqrt{2}}\left[\begin{array}{cc}1 & 1 \\ 1 & -1\end{array}\right]$ be the familiar Hadamard matrix and define $H_{L}$ as its $L$-th tensor power, the matrix $L$ Hadamard transform of order $L$ :

$$
H_{L}=H^{\otimes L}=\frac{1}{2^{L / 2}}\left[(-1)^{\boldsymbol{b} \cdot \boldsymbol{c}}\right]_{\boldsymbol{b}, \boldsymbol{c} \in \mathbb{B}^{L}} .
$$

The columns of this matrix, denoted by $\left|w^{\boldsymbol{b}}\right\rangle=H_{L}|\boldsymbol{b}\rangle$ for $\boldsymbol{b} \in \mathbb{B}^{L}$, form an orthonormal basis $W_{L}=$ $\left\{H_{L}|\boldsymbol{b}\rangle ; \boldsymbol{b} \in \mathbb{B}^{L}\right\}$ of $\mathcal{V}_{L}$. The following assertion is easily checked:

Lemma 6 The (pairwise commuting) transformations $\sigma^{c}\left(\boldsymbol{c} \in \mathbb{B}^{L}\right)$ diagonalize in the $W_{L}$-basis. More precisely:

$$
\sigma^{\boldsymbol{c}}\left|w^{\boldsymbol{b}}\right\rangle=(-1)^{\boldsymbol{b} \cdot \boldsymbol{c}}\left|w^{\boldsymbol{b}}\right\rangle \quad\left(\boldsymbol{b}, \boldsymbol{c} \in \mathbb{B}^{L}\right)
$$


Thus also the transformation $\mathcal{A}_{L}$ diagonalizes in the $W_{L}$-basis and its eigenvalues are given by

$$
\left(H_{L} \cdot A_{L} \cdot H_{L}\right)\left|w^{\boldsymbol{b}}\right\rangle=\lambda_{\boldsymbol{b}}\left|w^{\boldsymbol{b}}\right\rangle
$$

where $\lambda_{\boldsymbol{b}}=\sum_{c \in \mathbb{B}^{L}} \alpha_{\boldsymbol{c}}(-1)^{\boldsymbol{b} \cdot \boldsymbol{c}}=\sum_{c \in \mathbb{B}^{L}} \alpha_{\boldsymbol{c}}\langle\boldsymbol{b}|H| \boldsymbol{c}\rangle$.

The crucial observation is now the following: even though the transformation $\mathcal{A}_{L}$ diagonalizes in the $W_{L}$-basis, the transformation $\mathcal{B}_{L}$ doesn't, it is not even triangular in this basis. But it turns out that a slight modification of the $W_{L}$-basis will be suitable for at the same time diagonalizing $\mathcal{A}_{L}$ and bringing the $\mathcal{B}_{L}$ in (lower) triangular form. For that purpose we introduce the invertible linear transformation

$$
\Delta: \mathbb{B}^{L} \rightarrow \mathbb{B}^{L}: \boldsymbol{b}=b_{1} b_{2} \ldots b_{L} \mapsto \boldsymbol{b}^{\Delta}=\left[\sum_{1 \leq i \leq L-j+1} b_{i}\right]_{1 \leq j \leq L}
$$

where the sum has to be taken in the binary field. As an example $(L=3)$ :

\begin{tabular}{c|cccccccc}
$\boldsymbol{b}$ & 000 & 001 & 010 & 011 & 100 & 101 & 110 & 111 \\
\hline $\boldsymbol{b}^{\Delta}$ & 000 & 100 & 110 & 010 & 111 & 011 & 001 & 101
\end{tabular}

The basis $\widetilde{W}_{L}=\left\{\left|w^{b^{\Delta}}\right\rangle\right\}$ is nothing but a rearrangement of the $W_{L}$-basis, hence the transformation $\mathcal{A}_{L}$ diagonalizes in this basis as well (with the corresponding eigenvalues). We will write $\widetilde{H}_{L}$ for the rearrangement of the Hadamard matrix in this new ordering of the elements of $\mathcal{B}^{L}$. The clue is now contained in the following proposition:

\section{Proposition 7}

$$
\widetilde{H}_{L} \cdot B_{L}(\boldsymbol{\beta}) \cdot \widetilde{H}_{L}=B_{L}^{\mathrm{t}}\left(\boldsymbol{\beta}^{\mathrm{rev}}\right)
$$

where $\boldsymbol{\beta}^{\text {rev }}=\left(\beta_{L}, \beta_{L-1}, \ldots, \beta_{1}\right)$ is the reverse of $\boldsymbol{\beta}=\left(\beta_{1}, \beta_{2}, \ldots, \beta_{L}\right)$, t denoting transposition.

We illustrate this proposition in the case $L=3$ by displaying matrices $2^{3 / 2} \widetilde{H}_{3}$ (left) and $\widetilde{H}_{3} \cdot B_{3}(\beta, \gamma, \delta)$. $\widetilde{H}_{3}=B_{3}(\delta, \gamma, \beta)^{\mathrm{t}}$ (right). Note that $\widetilde{H}_{L}$ is symmetric because $\Delta$ (as a matrix) is symmetric.

$$
\left[\begin{array}{rrrrrrrr}
1 & 1 & 1 & 1 & 1 & 1 & 1 & 1 \\
1 & 1 & 1 & 1 & -1 & -1 & -1 & -1 \\
1 & 1 & -1 & -1 & -1 & -1 & 1 & 1 \\
1 & 1 & -1 & -1 & 1 & 1 & -1 & -1 \\
1 & -1 & -1 & 1 & -1 & 1 & 1 & -1 \\
1 & -1 & -1 & 1 & 1 & -1 & -1 & 1 \\
1 & -1 & 1 & -1 & 1 & -1 & 1 & -1 \\
1 & -1 & 1 & -1 & -1 & 1 & -1 & 1
\end{array}\right],\left[\begin{array}{cccccccc}
0 & 0 & 0 & 0 & 0 & 0 & 0 & 0 \\
-\beta & \beta & 0 & 0 & 0 & 0 & 0 & 0 \\
0 & -\gamma & \gamma & 0 & 0 & 0 & 0 & 0 \\
-\gamma & 0 & -\beta & \beta+\gamma & 0 & 0 & 0 & 0 \\
0 & 0 & -\delta & 0 & \delta & 0 & 0 & 0 \\
0 & 0 & 0 & -\delta & -\beta & \beta+\delta & 0 & 0 \\
-\delta & 0 & 0 & 0 & 0 & -\gamma & \gamma+\delta & 0 \\
0 & -\delta & 0 & 0 & -\gamma & 0 & -\beta & \beta+\gamma+\delta
\end{array}\right] .
$$

The proof of Proposition 7 will be given below. It leads to the main result by looking at the matrix representation of $\mathcal{M}_{L}(\boldsymbol{\alpha}, \boldsymbol{\beta})=\mathcal{A}_{L}-\mathcal{B}_{L}$ in the $\widetilde{W}_{L}$-basis, where it takes lower triangular form. Hence the eigenvalues, which are $2^{L}$ pairwise distinct linear polynomials in the $\alpha$ - and $\beta$-variables, can be read directly from the main diagonal. In contrast to Theorem 4 , the ex-conjecture, all eigenvalues are simple. 


\section{Theorem 8}

$$
\operatorname{det} M_{L}(\boldsymbol{\alpha}, \boldsymbol{\beta})=\operatorname{det}\left[A_{L}(\boldsymbol{\alpha})-B_{L}(\boldsymbol{\beta})\right]=\prod_{\boldsymbol{b} \in \mathbb{B}^{L}}\left(\lambda_{\boldsymbol{b}^{\Delta}}-\boldsymbol{\beta}^{\mathrm{rev}} \cdot \boldsymbol{b}\right)
$$

Illustration of the Theorem for $L=3$ recalling $\boldsymbol{\beta}=(\beta, \gamma, \delta)=\left(\beta_{1}, \beta_{2}, \beta_{3}\right)$ :

$\begin{array}{cccl}\boldsymbol{b} & \boldsymbol{b}^{\Delta} & \lambda_{b^{\Delta}} & (\delta, \gamma, \beta) \cdot \boldsymbol{b} \\ 000 & 000 & {[++++++++] \cdot \boldsymbol{\alpha}} & 0 \\ 001 & 100 & {[++++----] \cdot \boldsymbol{\alpha}} & \beta \\ 010 & 110 & {[++----++] \cdot \boldsymbol{\alpha}} & \gamma \\ 011 & 010 & {[++--++--] \cdot \boldsymbol{\alpha}} & \beta+\gamma \\ 100 & 111 & {[+--+-++-] \cdot \boldsymbol{\alpha}} & \delta \\ 101 & 011 & {[+--++--+] \cdot \boldsymbol{\alpha}} & \beta+\delta \\ 110 & 001 & {[+-+-+-+-] \cdot \boldsymbol{\alpha}} & \gamma+\delta \\ 111 & 101 & {[+-+--+-+] \cdot \boldsymbol{\alpha}} & \beta+\gamma+\delta\end{array}$

So, as an example, the line for $\boldsymbol{b}=101$ contributes the factor $\alpha_{000}-\alpha_{001}-\alpha_{010}-\alpha_{011}+\alpha_{100}-$ $\alpha_{101}-\alpha_{110}-\alpha_{111}-\beta-\delta$ to the product.

To prepare for the proof of Proposition 7 we state without proof simple relations between the transformations $\psi_{j}, \phi_{L-j+1}$ and $\Delta$ :

Lemma 9 For $\boldsymbol{b}, \boldsymbol{c} \in \mathbb{B}^{L}$ and $1 \leq j \leq L$ we have

1. $\left(\psi_{j} \boldsymbol{b}\right)^{\Delta}=\phi_{L-j+1}\left(\boldsymbol{b}^{\Delta}\right)$

2. $\boldsymbol{b}^{\Delta} \cdot \psi_{j} \boldsymbol{c}=\boldsymbol{b}^{\Delta} \cdot \boldsymbol{c}+b_{L-j+1}$

Fact 2. is a consequence of fact 1 .

Proof of Proposition 7 The actions of the transformations $\mathcal{P}_{L, j}$, seen in the $\widetilde{W}_{L}$-basis, are given by:

$$
\mathcal{P}_{L, j}:\left|w^{\boldsymbol{b}^{\Delta}}\right\rangle \mapsto \begin{cases}-\left|w^{\left(\psi_{L-j+1} \boldsymbol{b}\right)^{\Delta}}\right\rangle & \text { if } b_{L-j+1}=0 \\ \left|w^{\boldsymbol{b}^{\Delta}}\right\rangle & \text { if } b_{L-j+1}=1 .\end{cases}
$$

To see this, we compute

$$
\begin{aligned}
\mathcal{P}_{L, j}\left|w^{\boldsymbol{b}^{\Delta}}\right\rangle & =\sum_{\boldsymbol{c} \in \mathbb{B}^{L}}\left\langle\boldsymbol{b}^{\Delta}|H| \boldsymbol{c}\right\rangle \mathcal{P}_{L, j}|\boldsymbol{c}\rangle=\sum_{\boldsymbol{c}>\psi_{j} \boldsymbol{c}}\left\langle\boldsymbol{b}^{\Delta}|H| \boldsymbol{c}\right\rangle\left(|\boldsymbol{c}\rangle-\left|\psi_{j} \boldsymbol{c}\right\rangle\right) \\
& =\sum_{\boldsymbol{c}>\psi_{j} \boldsymbol{c}}\left\langle\boldsymbol{b}^{\Delta}|H| \boldsymbol{c}\right\rangle|\boldsymbol{c}\rangle-\sum_{\boldsymbol{c}<\psi_{j} \boldsymbol{c}}\left\langle\boldsymbol{b}^{\Delta}|H| \psi_{j} \boldsymbol{c}\right\rangle|\boldsymbol{c}\rangle
\end{aligned}
$$

using the involutive nature of $\psi_{j}$ for the second sum. Now, using 2. from Lemma9,

$$
\left\langle\boldsymbol{b}^{\Delta}|H| \psi_{j} \boldsymbol{c}\right\rangle=(-1)^{\boldsymbol{b}^{\Delta} \cdot \psi_{j} \boldsymbol{c}}=(-1)^{\boldsymbol{b}^{\Delta} \cdot \boldsymbol{c}+b_{L-j+1}}=(-1)^{b_{L-j+1}}\left\langle\boldsymbol{b}^{\Delta}|H| \boldsymbol{c}\right\rangle
$$

and thus

$$
\mathcal{P}_{L, j}\left|w^{\boldsymbol{b}^{\Delta}}\right\rangle=\sum_{\boldsymbol{c}>\psi_{j} \boldsymbol{c}}\left\langle\boldsymbol{b}^{\Delta}|H| \boldsymbol{c}\right\rangle|\boldsymbol{c}\rangle-(-1)^{b_{L-j+1}} \sum_{\boldsymbol{c}<\psi_{j} \boldsymbol{c}}\left\langle\boldsymbol{b}^{\Delta}|H| \boldsymbol{c}\right\rangle|\boldsymbol{c}\rangle .
$$


The conclusion in the case $b_{L-j+1}=1$ is now obvious.

As for the case $b_{L-j+1}=1$, we see, using item 1. from Lemma9, that

$$
\begin{aligned}
\left|w^{\left(\psi_{L-j+1} \boldsymbol{b}\right)^{\Delta}}\right\rangle & =\sum_{\boldsymbol{c} \in \mathbb{B}^{L}}\left\langle\left(\psi_{L-j+1} \boldsymbol{b}\right)^{\Delta}|H| \boldsymbol{c}\right\rangle|\boldsymbol{c}\rangle=\sum_{\boldsymbol{c} \in \mathbb{B}^{L}}(-1)^{\left(\psi_{L-j+1} \boldsymbol{b}\right)^{\Delta} \cdot \boldsymbol{c}}|\boldsymbol{c}\rangle \\
& =\sum_{\boldsymbol{c} \in \mathbb{B}^{L}}(-1)^{\phi_{j}\left(\boldsymbol{b}^{\Delta}\right) \cdot \boldsymbol{c}}|\boldsymbol{c}\rangle=\sum_{\boldsymbol{c} \in \mathbb{B}^{L}}(-1)^{\boldsymbol{b}^{\Delta} \cdot \boldsymbol{c}+c_{j}}|\boldsymbol{c}\rangle \\
& =-\sum_{\boldsymbol{c}: c_{j}=1}\left\langle\boldsymbol{b}^{\Delta}|H| \boldsymbol{c}\right\rangle|\boldsymbol{c}\rangle+\sum_{\boldsymbol{c}: c_{j}=0}\left\langle\boldsymbol{b}^{\Delta}|H| \boldsymbol{c}\right\rangle|\boldsymbol{c}\rangle=-\mathcal{P}_{L, j}\left|w^{\boldsymbol{b}^{\Delta}}\right\rangle .
\end{aligned}
$$

Corollary 10 If we consider the special case where $\alpha_{\boldsymbol{b}}=0$ for all $\boldsymbol{b} \in \mathbb{B}^{L}$, except $\alpha_{00 \ldots 0}=\alpha_{0}$ and $\alpha_{10 \ldots 00}=\alpha_{1}$, and where $\beta_{1}=\ldots=\beta_{L-1}=1$ and $\beta_{L}=\beta$, then the determinant of the Theorem simplifies to the product $\Pi_{1} \cdot \Pi_{2} \cdot \Pi_{3} \cdot \Pi_{4}$ of the following four terms:

$$
\begin{array}{ll}
\Pi_{1}=\prod_{0 \leq 2 k<L}\left(\alpha_{0}+\alpha_{1}-2 k\right)^{\left(\begin{array}{c}
L-1 \\
2 k
\end{array}\right)} & \Pi_{2}=\prod_{0 \leq 2 k-1<L}\left(\alpha_{0}+\alpha_{1}-\beta-2 k+1\right)^{\left(\begin{array}{c}
L-1 \\
2 k-1
\end{array}\right)} \\
\Pi_{3}=\prod_{0 \leq 2 k-1<L}\left(\alpha_{0}-\alpha_{1}-2 k+1\right)^{\left(\begin{array}{c}
L-1 \\
2 k-1
\end{array}\right)} & \Pi_{4}=\prod_{0 \leq 2 k<L}\left(\alpha_{0}-\alpha_{1}-\beta-2 k\right)^{\left(\begin{array}{c}
L-1 \\
2 k
\end{array}\right)}
\end{array}
$$

For the proof note that each $\boldsymbol{b} \in \mathbb{B}^{L}$ we get as the contribution from $\mathcal{A}_{L}$

$$
\begin{aligned}
\lambda_{\boldsymbol{b}^{\Delta}} \cdot \boldsymbol{\alpha} & =\sum_{\boldsymbol{c} \in \mathbb{B}^{L}} \alpha_{\boldsymbol{c}}\left\langle\boldsymbol{b}^{\Delta}|H| \boldsymbol{c}\right\rangle \\
& =\alpha_{0}\left\langle\boldsymbol{b}^{\Delta}|H| 00 \ldots 00\right\rangle+\alpha_{1}\left\langle\boldsymbol{b}^{\Delta}|H| 10 \ldots 00\right\rangle \\
& =\alpha_{0}(-1)^{\boldsymbol{b} \cdot \Delta \cdot 00 \ldots 00}+\alpha_{1}(-1)^{\boldsymbol{b} \cdot \Delta \cdot 10 \ldots 00}=\alpha_{0}+(-1)^{\|\boldsymbol{b}\|} \alpha_{1}
\end{aligned}
$$

because $\Delta \cdot 00 \ldots 00=11 \ldots 11$ and then $\boldsymbol{b} \cdot 11 \ldots 11 \equiv\|\boldsymbol{b}\| \bmod 2$, where $\|\boldsymbol{b}\|$ denotes the Hamming weight of $\boldsymbol{b}$ and where we have used the fact that $\Delta$ is a symmetric matrix. Thus the $2^{L}$ eigenvalues are

$$
\alpha_{0}+(-1)^{\|\boldsymbol{b}\|} \alpha_{1}-\boldsymbol{\beta}^{\mathrm{rev}} \cdot \boldsymbol{b} \quad\left(\boldsymbol{b} \in \mathbb{B}^{L}\right) .
$$

Now there are four cases to consider:

1. $\|\boldsymbol{b}\|$ is even and $b_{1}=0$ : this gives eigenvalues $\alpha_{0}+\alpha_{1}-\left\|b_{2} b_{3} \ldots b_{L}\right\|$ and since $b_{1}$ does not contribute to $\|\boldsymbol{b}\|$ the vector $b_{2} b_{3} \ldots b_{L}$ must have even weight $2 k$. There are $\left(\begin{array}{c}L-1 \\ 2 k\end{array}\right)$ possibilities which account for $\Pi_{1}$.

2. $\|\boldsymbol{b}\|$ is even and $b_{1}=1$ : this gives eigenvalues $\alpha_{0}+\alpha_{1}-\beta-\left\|b_{2} b_{3} \ldots b_{L}\right\|$ and since $b_{1}$ does contribute to $\|\boldsymbol{b}\|$ the vector $b_{2} b_{3} \ldots b_{L}$ must have odd weight $2 k-1$. There are $\left(\begin{array}{c}L-1 \\ 2 k-1\end{array}\right)$ possibilities which account for $\Pi_{2}$.

3. $\|\boldsymbol{b}\|$ is odd and $b_{1}=0$ : this gives eigenvalues $\alpha_{0}-\alpha_{1}-\left\|b_{2} b_{3} \ldots b_{L}\right\|$ and since $b_{1}$ does not contribute to $\|\boldsymbol{b}\|$ the vector $b_{2} b_{3} \ldots b_{L}$ must have odd weight $2 k-1$. There are $\left(\begin{array}{c}L-1 \\ 2 k-1\end{array}\right)$ possibilities which account for $\Pi_{3}$. 
4. $\|\boldsymbol{b}\|$ is odd and $b_{1}=1$ : this gives eigenvalues $\alpha_{0}-\alpha_{1}-\beta-\left\|b_{2} b_{3} \ldots b_{L}\right\|$ and since $b_{1}$ does contribute to $\|\boldsymbol{b}\|$ the vector $b_{2} b_{3} \ldots b_{L}$ must have even weight $2 k$. There are $\left(\begin{array}{c}L-1 \\ 2 k\end{array}\right)$ possibilities which account for $\Pi_{4}$.

Corollary 11 Setting now $\alpha_{0}=-\alpha$ and $\alpha_{1}=\alpha$, i.e., specializing as in Lemma 5 gives for $\operatorname{det} M_{L}$ a product $\Pi_{1}^{\prime} \cdot \Pi_{2}^{\prime} \cdot \Pi_{3}^{\prime} \cdot \Pi_{4}^{\prime}$ of the following four terms:

$$
\begin{array}{ll}
\Pi_{1}^{\prime}=\prod_{0 \leq 2 k<L}(-2 k)^{\left(\begin{array}{c}
L-1 \\
2 k
\end{array}\right)} & \Pi_{2}=\prod_{0 \leq 2 k-1<L}(-\beta-2 k+1)^{\left(\begin{array}{c}
L-1 \\
2 k-1
\end{array}\right)} \\
\Pi_{3}=\prod_{0 \leq 2 k-1<L}(-2 \alpha-2 k+1)^{\left(\begin{array}{c}
L-1 \\
2 k-1
\end{array}\right)} & \Pi_{4}=\prod_{0 \leq 2 k<L}\left(-2 \alpha_{1}-\beta-2 k\right)^{\left(\begin{array}{c}
L-1 \\
2 k
\end{array}\right)}
\end{array}
$$

which are precisely the $2 L$ distinct eigenvalues of the original Conjecture.

\section{Concluding remarks}

We have been able to solve Ayyer and Mallick's conjecture about the eigenvalues of the asymmetric annihilation process by embedding it into a more general model and using an orthogonal transform which triagonalizes the transition matrix. In sharp contrast to the original problem, the general situation with parameters $\alpha_{\boldsymbol{b}}$ and $b_{j}$ (which may be given a "physical" interpretation using $\left\langle\boldsymbol{b}\left|A_{L}\right| \boldsymbol{c}\right\rangle=\alpha_{\boldsymbol{b} \oplus \boldsymbol{c}}$ and (17, (2)) is easier to handle because it is not degenerate: all "symbolic" eigenvalues are simple. Our proof does not seem to explain the maximum amount of degeneracy, as stated in Conjecture 1.

On the other hand, we mention that the result of Corollary 3 about the partition function can be extended to the more general model. Again, in contrast to the inductive approach of Ayyer and Mallick in [2], as outlined in Sec. 3, we can solve this problem directly by transforming it orthogonally into the basis where it shows its triangular structure.

We start by remarking that the columns sums of the extended model are constant $\sum \alpha_{c}$, though not zero. This implies that $\left\langle 1_{L}\right|$ is the unique left eigenvector with eigenvalue $\bar{\alpha}=\sum \alpha_{c}$ of $M_{L}(\alpha, \beta)$. The right eigenvector $|\boldsymbol{x}\rangle$ with the same eigenvalue corresponds to the steady state distribution of the original problem. Then $|\boldsymbol{y}\rangle=\widetilde{H}_{L}|\boldsymbol{x}\rangle$ satisfies $\widetilde{M}_{L}(\boldsymbol{\alpha}, \boldsymbol{\beta})|\boldsymbol{y}\rangle=\bar{\alpha}|\boldsymbol{y}\rangle$, where $\widetilde{M}_{L}=\widetilde{H}_{L} \cdot M_{L}(\boldsymbol{\alpha}, \boldsymbol{\beta}) \cdot \widetilde{H}_{L}$ is the matrix seen in the $\widetilde{W}_{L}$-basis. This triangular system for $y$ is written explicitly as

$$
\left(\lambda_{b^{\Delta}}^{*}+\boldsymbol{\beta}^{\mathrm{rev}} \cdot \boldsymbol{b}\right) y_{\boldsymbol{b}}=\sum_{j: b_{j}=1} \beta_{L-j+1} y_{\psi_{j} \boldsymbol{b}} \quad\left(\boldsymbol{b} \in \mathbb{B}^{L}\right)
$$

where

$$
\lambda_{\boldsymbol{b}}^{*}=\bar{\alpha}-\lambda_{\boldsymbol{b}}=2 \sum_{\boldsymbol{c}: \boldsymbol{b} \cdot \boldsymbol{c}=1} \alpha_{\boldsymbol{c}} \quad\left(\boldsymbol{b} \in \mathbb{B}^{L}\right) .
$$

Note that the sum on the right only contains terms $y_{\boldsymbol{c}}$ where $\boldsymbol{c}=\psi_{j} \boldsymbol{b}<\boldsymbol{b}$. For $\boldsymbol{b}=00 \ldots 0$ the equation is void, so we may put $y_{00 \ldots 0}=1$. Since the polynomials $\lambda_{b \Delta}^{*}+\boldsymbol{\beta}^{\text {rev }} \cdot \boldsymbol{b}$ are mutually coprime, this shows by induction that the denominator of the rational normal form of $y_{b}$ is the product of all polynomials $\lambda_{c^{\Delta}}^{*}+\boldsymbol{\beta}^{\text {rev }} \cdot \boldsymbol{c}$, where $\boldsymbol{c}$ runs over the binary vectors that can be obtained from $\boldsymbol{b}$ by successive application of decreasing $\psi_{j}$-transformations. Consequently, the product of linear polynomials

$$
Z(\boldsymbol{\alpha}, \boldsymbol{\beta})=\prod_{\mathbf{0} \neq \boldsymbol{b} \in \mathbb{B}^{L}}\left(\lambda_{b^{\Delta}}^{*}+\boldsymbol{\beta}^{\mathrm{rev}} \cdot \boldsymbol{b}\right)
$$


is the least common multiple of the denominators of the $y_{\mathbf{b}}$. This property is invariant under the Hadamard transform, so it applies also to the coefficients of $|\boldsymbol{x}\rangle=\widetilde{H}_{L}|\boldsymbol{y}\rangle$. But

$$
\left\langle\mathbf{1}_{L} \mid \boldsymbol{x}\right\rangle=\left\langle\mathbf{1}_{L}\left|\widetilde{H}_{L}\right| \boldsymbol{y}\right\rangle=2^{L / 2}\langle 100 \ldots 00 \mid \boldsymbol{y}\rangle=2^{L / 2} y_{00 \ldots 0}=2^{L / 2},
$$

so $2^{-L / 2}|\boldsymbol{x}\rangle$ is already normalized and can be seen as the "symbolic" stationary distribution in the generalized model. What we have shown is:

Theorem $12 Z(\boldsymbol{\alpha}, \boldsymbol{\beta})$ is the partition function related to $M_{L}(\boldsymbol{\alpha}, \boldsymbol{\beta})$.

We conclude by remarking that the specialization as in Lemma 5 and Corollary 11 brings us back to Corollary 3 . This is not completely obvious, since the expression in Corollary 3 has only $\left(\begin{array}{c}L+1 \\ 2\end{array}\right)$ factors, whereas in Theorem 12 there are $2^{L}-1$ factors. What happens is that upon specialization the requirements for least common multiples and greatest common divisors change. Taking this into account one finds that from the general expression for $Z(\boldsymbol{\alpha}, \boldsymbol{\beta})$ only the $\left(\begin{array}{c}L+1 \\ 2\end{array}\right)$ terms where $\boldsymbol{b} \in \mathbb{B}^{L}$ with $\|\boldsymbol{b}\|=1$ or 2 contribute - and this is precisely the statement of Corollary 3 .

\section{References}

[1] B. Derrida, M. R. Evans, V. Hakim, V. Pasquier, Exact solution of a 1D asymmetric exclusion model using a matrix formulation, J. Phys. A: Math. Gen. 26 (1993), 1493-1517.

[2] A. Ayyer and K. Mallick, Exact results for an asymmetric annihilation process with open boundaries, J. Phys. A: Math. Gen. 43 (2010) 045033, 22pp.

[3] L. Shapiro and D. Zeilberger, A Markov chain occurring in Enzyme Kinetics, J. Math. Biology 15 (1982) 351-357.

[4] E. Duchi and G. Schaeffer, A combinatorial approach to jumping particles: The parallel TASEP, Random Structures and Algorithms, 33 no. 4 (2008),434-451.

[5] Richard Brak, S. Corteel, J. Essam R. Parviainen and A. Rechnitzer, A Combinatorial Derivation of the PASEP Stationary State, Electron. J. Combin. 13 no. 1 (2006), 108, 23 pp.

[6] S. Corteel and L. Williams, Tableaux combinatorics for the asymmetric exclusion process, Advances in Applied Mathematics, 39 no. 3 (2007), 293-310.

[7] S. Corteel and L. Williams, A Markov chain on permutations which projects to the PASEP, International Mathematics Research Notices, 2007 (2007) rnm055, 27 pp.

[8] S. Corteel and L. Williams, Staircase tableaux, the asymmetric exclusion process and Askey-Wilson polynomials, to appear in Proc. Nat. Acad. Sci. USA, arXiv: 0910.1858.

[9] G. X. Viennot, Canopy of binary trees, Catalan tableaux and the asymmetric exclusion process, Proceedings of FPSAC 2007, Tianjin, China, preprint, arXiv: 0905.3081.

[10] S. Corteel, M. Josuat-Verges, T. Prellberg and M. Rubey, Matrix Ansatz, lattice paths and rook placements, Proceedings of FPSAC2009, DMTCS proc. AK, 104 (2009), 313-324, arXiv: 0811.4606. 